\title{
A synthetic indicator to evaluate the contribution of clean development mechanism projects for Latin American sustainable development
}

\author{
H. F. Beirão Junior \\ Federal Institute of Education, Science and Technology of \\ Santa Catarina, Campus Florianopolis, Brazil
}

\begin{abstract}
If one were to assign responsibilities of current climate change, one of the most important challenges of the 21 st century, it probably does not correspond to the Latin American countries, which are most vulnerable to the effects of greenhouse gas emission (GHG). One of the opportunities to meet the challenge of climate change is already being implemented, because the so-called rich countries are developing environmentally sustainable projects in Latin America to account for their own GHG reductions generated in the region of developing countries who ratified the Kyoto Protocol. Therefore, the Clean Development Mechanism (CDM), a flexibility mechanism established by Article 12 of the Kyoto Protocol, constitutes a real opportunity for developing countries to achieve the benefits of the emerging carbon market. Not everything can be considered a benefit to the host countries of these CDM projects as there is a risk that they are better adapted to the economic interests of the industrialized country than the host country. This paper proposes the creation of a synthetic indicator to quantitatively measure and summarize the contribution of CDM projects on complex phenomenon such as sustainable development in a particular region. It is expected that the Sustainable Development Index of Project (SDIP) serves as a monitoring mechanism for economic, environmental, and social impacts, and supports decision-making for the validation of a CDM project. The simplification suggested by the SDIP is important because it can provide an easily identifiable numerical value which represents the CDM project's viability.
\end{abstract}


Moreover, the degree of standardization of the proposed index in relation to CDM projects evaluated allows for comparison, so those projects with the highest rates are the projects selected for implementation in the host regions.

Keywords: climate change, clean development mechanism project, sustainable development, synthetic index.

\section{Introduction}

Global climate change is one of the most important challenges of the 21st century and its impacts may be irreversible. Island countries and cities located in coastal areas are most vulnerable to climate changes, with the possibility of flooding over the medium and long term.

The Fourth Assessment Report [2] provides some examples of projected impacts on different regions - probably some systems, sectors, and regions will be particularly affected by climate change. In Latin America, one of the regions with lower emissions of greenhouse gases (GHG), but which countries are most vulnerable to the effects of these gases, the report stated:

- By mid-century, increases in temperature and associated decreases in soil moisture originate a gradual replacement of tropical forest by savanna in eastern Amazonia. Semi-arid vegetation would be replaced by arid-land vegetation.

- A significant loss of biodiversity could be experienced with the extinction of species in many areas of tropical Latin America.

- Productivity of some important crops would decline, and with it livestock productivity, with adverse consequences for food security. In temperate zones soybean crop yields would improve. Taken together, they could increase the number of people threatened by famine.

- Changes in rainfall patterns and the disappearance of glaciers would markedly impair the availability of water for human consumption, agriculture, and hydropower.

\section{The clean development mechanism}

In a global strategy to combat climate change, the so-called rich countries are developing environmentally sustainable projects in Latin America to account as their own GHG reductions generated in the region of the developing countries which ratified the Kyoto Protocol. Thus, the Clean Development Mechanism (CDM) under Article 12 of the Kyoto Protocol can be considered to be a main instrument for developing country participation on issues related to climate change. Through the same mechanism, countries listed in Annex B [3] of the Protocol, or companies based on them, can invest in carbon emission reduction or retention (sequestration) of projects in developing countries.

Although recent years have shown that CDM are real opportunities for developing countries to achieve the benefits of the emerging carbon market, not everything can be considered a benefit to these CDM project host countries as 
there is a risk that they are better adapted to the industrialized country's economic interests than those of the host country. The dual objective requires that CDM projects should contribute to the sustainable development of the countries in which they are enacted, in addition to reducing GHG emissions. However, some studies show that this instrument contributes little to the host country's development, with the specific impact on local communities even more uncertain and sometimes negative.

This is due to the absence of a policy framework or inclusion procedure in analyzing CDM projects on their impact upon the socioeconomic development of communities within their area of influence. Such a framework would allow us to evaluate the commitments made by the project's promoters in terms of sustainable development and especially the development of the poorest communities. In practice, a project can be registered without having to show any positive impact on sustainable development beyond the emissions reduction, even while having negative impacts on local communities [4].

\section{The sustainable development index of project}

This paper presents a proposal to create a synthetic indicator to quantitatively measure and summarize CDM project contribution to complex phenomenon such as the sustainable development of a particular region. It is expected that the Sustainable Development Index of Project (SDIP) serves as a monitoring mechanism for the environmental, economic, and social impacts, and supports decision-making in CDM project validation. It is intended thereby to prevent seemingly interesting projects for implementation in Latin American countries which may contain more weaknesses than strengths from the Latin American perspective.

Thus, the Sustainable Development Index of Project (SDIP) is obtained from three composite or synthetic indicators representing the environmental, economic, and social dimensions of a CDM project's contribution to sustainable development. The development of this index is based on the methodology for calculating the United Nations Human Development Index (HDI) [5], although the environmental, economic, and social components replace health, education, and standard of living in the HDI.

The simplification proposed for the SDIP is important because it can provide a readily identifiable numeric value to represent the feasibility or impracticability of the proposed CDM project. Another application for the index is the possibility to evaluate already implemented projects in order to determine whether these projects are achieving their objectives in relation to their impact on sustainable development in the host country region.

Similar to that used in the current HDI, the SDIP also uses the geometric mean, which unlike the arithmetic mean penalizes the fact that the environmental, economic, and social component indexes are very different from each other. That is, to improve the SDIP it would be desirable to improve the 
rates of the three components, not just one. Furthermore, it is assumed that these components are not replaceable, in other words, it matters that the three components (environmental, economic, and social) are highly valued and developed in the CDM project.

The proposed development of a composite indicator through this study aims to identify and evaluate concepts from the design document of a CDM project if it includes the criteria that are part of the SDIP, and to get the quantity or quality of such criteria presented in order to calculate the SDIP of the CDM project.

The novelty of this approach lies in the quantitative expression of the CDM project's contribution to sustainable development, which comprises the three components or dimensions mentioned above. Each of them may also be separately quantified from a set of baseline indicators. Thus, it is possible to create a measurable, clear, and transparent expression.

In order to establish criteria for the creation of the proposed index it was necessary to select the baseline indicators which most significantly contribute to sustainable development. To do this, some of the relevant research findings made by international research institutions and the indicators currently included in government agency indicator lists were used.

For the resulting value of the SDIP, the composite index for each dimension considered was first calculated. Each had the same participation towards the final composition of SDIP:

a) Environmental Synthetic Index of Project (EnSIP) - consists of the following baseline indicators: reducing GHG emissions, corresponding to $40 \%$ of the index; reducing the level of air pollution (non-GHG emissions), corresponding to $15 \%$ of the index; reducing the level of water pollution, for another $15 \%$ of the index; reducing the level of soil contamination, also corresponding to $15 \%$ of the index; and biodiversity protection, for the remaining $15 \%$.

b) Economic Synthetic Index of Project (EcSIP) - based on baseline indicators such as: employment generation, weighing a third of the total index; the generation and increased income earned by workers, with a weight of another third of the index; and technology transfer, for the last third of the EcSIP.

c) Social Synthetic Index of Project (SoSIP) - composed of the baseline indicators: the contribution of access to health, with a weight of $30 \%$; the contribution of access to education, also with a weight of $30 \%$; the contribution to energy access, with a weight of $20 \%$; the contribution of access to garbage collection and transportation, with a weight of $10 \%$; and the contribution of access to sanitation, also with a weight of $10 \%$.

The methodology used in this process of creating synthetic or compound indexes in generating SDIP is represented through a pyramidal structure where the base, much wider than the other levels, demonstrates how the thirteen baseline indicators are positioned and their importance towards the development of the SDIP (Fig. 1). 


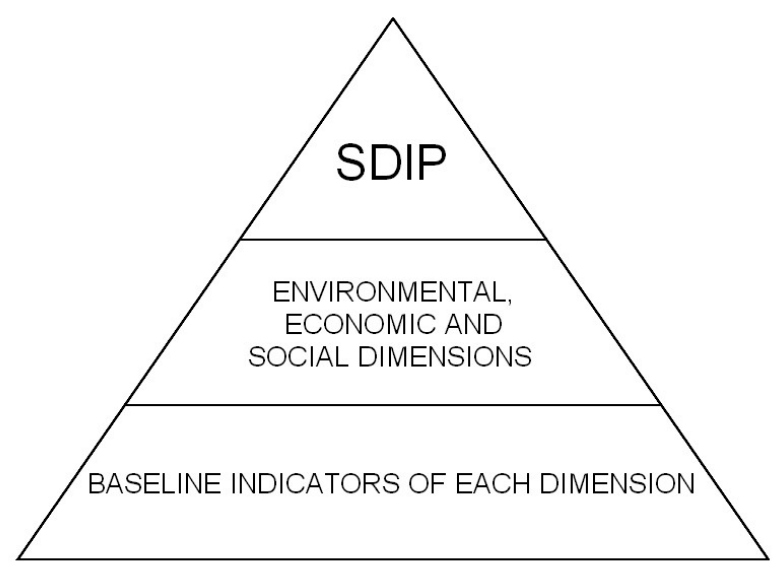

Figure 1: $\quad$ Pyramidal structure of SDIP construction [6].

The criteria or baseline indicators mentioned above proposed for consideration in assessing the impact of the CDM project and SDIP generation, based on known empirical knowledge, may have the following parameters to be expressed in a way that permits their aggregation:

a) Total emissions reduction of the six main greenhouse gases which contribute to greenhouse gases $\left(\mathrm{CO}_{2}, \mathrm{CH}_{4}, \mathrm{~N}_{2} \mathrm{O}, \mathrm{HFC}, \mathrm{PFC}\right.$, and $\left.\mathrm{SF}_{6}\right)$, commonly expressed as the percentage of $\mathrm{CO} 2$ equivalent reduction emitted into the atmosphere.

b) Reduction of air pollution, represented as the percentage of the reduction of air emissions of pollutants other than greenhouse gases which affect air quality.

c) Reduction of water pollution, expressed as the percentage of improvement of the quality or the percentage of increased distribution measured in $\mathrm{hm}^{3}$.

d) Reducing the level of soil contamination, represented by the percentage of improvement in quality or the percentage of improvement of soil surface affected by contamination.

e) Protection of biodiversity, expressed as the percentage of improvement in the number of species and habitats affected and/or threatened or the percentage of increased protected areas in the host region.

f) Generation of employment, expressed as the percentage of increase in the numbers of jobs directly and indirectly generated by the project compared to the local average.

g) Generation and increased income earned by the workers, represented by the percentage of the number of new businesses in the project area generated by the project, compared with the local business average generated in an equivalent period.

h) Technology transfers, expressed as the percentage rate of increase in local community training activities (workshops, seminars, and training activities) or percentage of increase in technology spending to the region hosting the project. 
i) Contribution of access to health represented by the percentage rate of increase of the local population with access to basic health care services.

j) The contribution of access to education, expressed as the percentage of change in access to educational activities of the local population.

k) Contribution to access to energy, represented by the percentage rate of increase of local population, in the project area, which has access to energy.

1) Contribution to access to waste collection and transportation, expressed as the percentage of increase in number of households in the project area with access to household waste collection.

m) Contribution of access to sanitation, as a percentage of the increased number of people from the local population with access to sanitation.

Note that in four of the thirteen baseline indicators there are two options of possible parameters included for testing the project's contribution to sustainable development of the region. This has occurred due to difficulties in obtaining data for these indicators. It should be noted that this complication could affect the results of the index if it is not possible to include them in the CDM project evaluated.

The values of the three synthetic indexes comprising the SDIP are between 0 and 1 . Therefore, the value of SDIP also varies from 0 to 1 .

\section{Conclusions}

The closer the value of SDIP approximates 1, the higher is its level of contribution to sustainable development in the region of the CDM project. Thus, the degree of SDIP standardization in connection with CDM projects was evaluated through comparison. The projects with the highest rates were selected for implementation in the host regions.

Note also that the preparation of a basis for a comparison in a given region, must take into account the main indicators available for the region. The SDIP assigned to the project must exceed the threshold for the host country region in order to be considered as a CDM project that really meets the requirements of the contribution to sustainable development. The threshold setting is not part of the objectives of this work and the construction of this threshold can be considered as an open line of research for future work.

Finally, it is important to note that the results of the proposed SDIP should be considered as relative measurements of sustainable CDM project performance and as a tool to highlight environmental, economic, and social issues which need exposure. The resulting assessment is subject to a number of uncertainties and qualifications, and therefore it has to express limits on the possibility of obtaining accurate conclusions because there are obstacles such as errors in defining indicators and a lack of data specified by the criteria which are compatible with those used in preparing the SDIP. 


\section{References}

[1] Beirão Junior, H.F., Study of economic and environmental indicators for the viability of the clean development mechanism in Latin America. Doctoral Thesis: University of Alcalá, Alcalá de Henares (Madrid), Spain, 2011.

[2] IPCC, Climate Change 2007 - Synthesis Report, Contribution of Working Groups I, II and III to the Fourth Assessment Report of Intergovernmental Panel on Climate Change: Geneva, Switzerland, 2007.

[3] United Nations, Kyoto Protocol to the United Nations Framework on Climate Change. New York, NY, USA, Annex B, 1998.

[4] Intermón Oxfam, CDM project impact on human development. Analysis of experiences in Morocco, Guatemala and Mexico. Intermón Oxfam Research Report. Madrid, Spain, 2009.

[5] United Nations Development Programme (UNDP). Human Development Index (HDI). www.hdr.undp.org/en/statistics/hdi

[6] Savings Banks Foundation, Equal opportunities in Spain, CECA / FUNCAS: Madrid, Spain, p. 75, 2011. 